\title{
Advanced Hepatic Fibrosis in Fatty Liver Disease Linked to Hyperplastic Colonic Polyp
}

\author{
Mahmud Mahamid, ${ }^{1,2}$ Omar Abu-Elhija, ${ }^{1,2}$ Tarik Yassin, ${ }^{3}$ and William Nseir ${ }^{2,4}$ \\ ${ }^{1}$ Internal Medicine Department, Holy Family Hospital, Nazareth, Israel \\ ${ }^{2}$ Faculty of Medicine in the Galilee, Bar-Ilan University, Safed, Israel \\ ${ }^{3}$ Internal Medicine Department, The Baruch Padeh Medical Center, Poriya, Israel \\ ${ }^{4}$ Internal Medicine Department, EMMS the Nazareth Hospital, Nazareth, Israel
}

Correspondence should be addressed to Mahmud Mahamid; mahmudmahamid@yahoo.com

Received 20 October 2016; Accepted 29 November 2016; Published 3 January 2017

Academic Editor: Mark Borgaonkar

Copyright (C) 2017 Mahmud Mahamid et al. This is an open access article distributed under the Creative Commons Attribution License, which permits unrestricted use, distribution, and reproduction in any medium, provided the original work is properly cited.

\begin{abstract}
Aim. Our study aims to determine possible association between biopsy-proven nonalcoholic steatohepatitis (NASH) and hyperplastic polyps (HP) of the colon. Methods. A retrospective cohort observational study. All subjects underwent screening colonoscopy within two years. Data were extracted from the patient charts including demographic, anthropometric measurement, vital signs, underlying diseases, medical therapy, laboratory data, results of the liver biopsy with degree of fibrosis and necroinflammatory activity, the colonoscopy report, and the pathological report of the extracted polyp. Results. A total of 223 patients were included in our study, 123 patients with biopsy-proven NASH and 100 patients without NASH who served as the control group matched for age. 14 colonic adenomas (11\% of patients) were found in the NASH group compared with 16 adenomas ( $16 \%$ of patients) found in the control group $(P=0.9) .28$ HPs were found in the NASH group $(22.7 \%)$ compared with only 8 HPs in the control group $(8 \%)$ $(P<0.05) .21$ from the $28(75 \%)$ HPs diagnosed in the NASH group were observed in the high degree fibrosis patients (Fibrosis Stages 3 and 4), 6 HPs (21\%) were associated with Fibrosis Stages 1 and 2, and single HP (4\%) was associated with Fibrosis Stage 0 . Conclusions. Our study showed an association between biopsy-proven steatohepatitis and the burden of hyperplastic polyp. The severity of hepatic fibrosis may play important role in the increased occurrence of HPs.
\end{abstract}

\section{Introduction}

Despite being the most common type of polyp detected in the human colon and rectum, relatively little is known about the etiology, natural history, or growth of hyperplastic polyps (HP) $[1,2]$.

HPs are considered benign lesions that have little or no malignant potential. However, recent studies have suggested that HP may either lie in the classic adenoma-carcinoma pathway $[3,4]$.

Lifestyle and dietary risk factors are the most common risk factors for development of HP according to epidemiological studies; other risk factors include alcohol consumption, cigarette smoking, obesity, metabolic syndrome, and fiber intake $[5,6]$.
Nonalcoholic fatty liver disease (NAFLD) is emerging condition and constituted an important public health problem across the globe. Fatty liver disease can present as simple hepatic steatosis that may progress to nonalcoholic steatohepatitis (NASH), fibrosis, cirrhosis, and hepatocellular carcinoma (HCC).

NAFLD is the most common cause of incidental elevated liver enzymes in the developed world. The prevalence of NASH in Europe and USA ranges from 14 to $20 \%$; this increase in the prevalence is related directly to obesity epidemically seen in these populations [7].

Diabetes mellitus (DM), obesity, and hyperlipidemia are common components of the metabolic syndrome (MS), which is frequently associated with the NAFLD. Therefore, NAFLD is the hepatic manifestation of MS [8]. 
The pathogenesis of NAFLD is not clearly elucidated, but accumulating data suggest that insulin resistance (IR), oxidative stress, lipotoxicity, intestinal endotoxins, and bacterial translocation which all related to the MS play a crucial role in the pathogenesis of steatosis, steatohepatitis, and fibrosis [9].

Although many studies have proposed that MS is a risk factor for colorectal cancer (CRC), colonic adenomas, hyperplastic polyps, and NAFLD [10], there have been no studies that investigate an association of hyperplastic polyps and NAFLD. Therefore, we investigated whether a relationship of HP to NAFLD could be determined.

\section{Methods}

A retrospective cohort observational study was conducted at Division of Internal Medicine of the Holy Family Hospital, Nazareth, Israel, between April 2013 and April 2015 on patients who underwent screening colonoscopy.

The study was approved by the local ethics committee. The data were coded to keep anonymity of the patients. Informed consent was waived because of the noninterventional study design.

2.1. Patient Selection and Data Collection. The study population of this study consisted of adult patients with biopsyproven NASH who underwent a screening colonoscopy in the past two years. Subjects were followed up at the Internal Medicine Department of the Holy Family Hospital (HFH). Patients were included only if they had underwent a screening colonoscopy at our facility within 2 years and underwent liver biopsy in the past 5 years.

2.2. Exclusion Criteria Included. NASH patients without biopsy performed to confirm the diagnosis and patients with other liver diseases, with known colonic disease including inflammatory bowel disease and polyposis syndrome, with history of total or segmental colectomy, with family or personal history of colonic cancer or colonic polyps, and with sessile serrated adenomas. The control group consisted with non-NASH patients, matched in age.

Data were obtained from the medical charts of all enrolled patients and from family physician especially in case that there was any missing data for study purposes. The following information was extracted from patient's charts: demographic, anthropometric measurement, vital signs, underlying diseases, medical therapy, laboratory data, and result of the liver biopsy with degree of fibrosis and necroinflammatory activity, the colonoscopy report, and the pathological report of the extracted polyp.

2.3. Measurements. The body mass index (BMI) for all patients was calculated in $\mathrm{kg} / \mathrm{m}^{2}$. A single expert hepatopathologist reviewed all liver biopsies and utilized the scoring system established by Brunt and colleagues [11] for grading and staging of steatohepatitis (staging of fibrosis is as follows: Stage 0, no fibrosis, Stage 1, zone 3 perisinusoidal fibrosis, Stage 2, as above with portal fibrosis, Stage 3, as above with bridging fibrosis, and Stage 4, cirrhosis).
TABLE 1: Demographic, laboratory, and clinical data comparing NASH group and control group.

\begin{tabular}{lccc}
\hline Characteristic & $\begin{array}{c}\text { Case } n=123 \\
(\%)\end{array}$ & $\begin{array}{c}\text { Control } n=100 \\
(\%)\end{array}$ & $P$ value \\
\hline Age (years) & $41 \pm 13$ & $42 \pm 12$ & NS \\
Male sex & $72(86 \%)$ & $43(43 \%)$ & $<0.05$ \\
BMI $\left(\mathrm{kg} / \mathrm{m}^{2}\right)$ & $25 \pm 4.7$ & $24.7 \pm 3.2$ & NS \\
Metabolic syndrome & $88(72 \%)$ & $57(57 \%)$ & NS \\
C-reactive protein & $1.1 \pm 0.7$ & $0.4 \pm 0.8$ & $<0.05$ \\
Ethnicity (Arabs) & $95(77 \%)$ & $72(72 \%)$ & NS \\
Diabetes mellitus & $37(30 \%)$ & $32(32 \%)$ & NS \\
Hypertension & $42(34 \%)$ & $39(39 \%)$ & NS \\
Hyperlipidemia & $49(40 \%)$ & $38(38 \%)$ & NS \\
Smoking & $8(15 \%)$ & $11(22 \%)$ & NS \\
\hline
\end{tabular}

2.4. Colonoscopy. Each patient underwent colonoscopy after completing a bowel preparation with 4 liter polyethylene glycol lavage solution; colonoscopies were performed by one of the five staff gastroenterologists at $\mathrm{HFH}$; each colonoscopy report was examined for the present of polyps, polyp location, polyp size, number of polyps, and histology; the location of polyp was divided into cecum, ascending, hepatic flexure, transverse, splenic flexure, descending, sigmoid, and rectal.

2.5. Statistical Analysis. Data were analyzed using SPSS version 19 (IBM SPSS, Chicago, IL, USA). Continuous variables are expressed as the mean \pm standard deviation. The Chi-square test was used to test differences in categorical variables between the cases and controls, and analysis of variance (ANOVA) or Student's $t$-test was used for comparisons of continuous variables. Spearman rank correlation and univariate regression analysis were used to determine the strength of the relationship between NAFLD and hyperplastic polyps after adjusting for independent variables previously known to be associated with HP, namely, age, gender, BMI, and diabetes mellitus. A multiple logistic regression analysis was done to determine the association between the different risk factors for HP. A significant level of $<0.05$ was used in this test.

\section{Results}

A total of 223 patients who underwent screening colonoscopy were included in our study, 123 patients with biopsy-proven NASH and 100 patients without NASH who served as the control group. Table 1 summarizes the clinical feature of the two groups, those with and without NAFLD.

Among the NASH group the 72 patients (86\%) were males; the mean age was $41 \pm 13$, the mean body mass index was $25.3 \pm 4.7$, patients who meet the criteria of metabolic syndrome were 88 patients (72\%), and the mean C-reactive protein level was $1.1 \pm 0.7$.

Regarding the polyps prevalence, 14 adenomas (11\% of patients) were found in the NASH group compared with 16 adenomas (16\% of patients) found in the control group $(P=$ $0.9)$. No significant differences were found when adjusting for 
TABLE 2: Multivariate analysis for the risk for hyperplastic polyp by gender, age, smoking, CRP level, prevalence of NASH, and the degree of liver fibrosis.

\begin{tabular}{lcc}
\hline Variable & OR $(95 \% \mathrm{CI})$ & $P$ value \\
\hline Male gender & $1.80(1.02-1.66)$ & $<0.001$ \\
Age $>50$ & $2.24(1.91-2.13)$ & 0.003 \\
$\mathrm{CRP}>2 \mathrm{mg} / \mathrm{L}$ & $1.47(1.14-2.18)$ & 0.027 \\
Current smoking & $1.35(1.05-1.67)$ & 0.022 \\
Prevalence of NASH & $1.69(1.36-198)$ & $<0.001$ \\
Fibrosis $>$ stage 2 & $1.82(1.23-1.76)$ & $<0.001$ \\
\hline
\end{tabular}

OR, odds ratio; CI, confidence interval; CRP, C-reactive protein.

known confounders to include age and BMI $(P=0.34)$. There was no significant increase in the risk for colonic adenomas when comparing individual component for MS and MS alone adenoma.

The location, size, number, morphology, and degree of dysplasia of the adenomas were similar between the two groups.

The prevalence of HP was statistically significant higher among the NASH group; $28 \mathrm{HPs}$ were found in the NASH group $(22.7 \%)$ compared with only 8 HPs in the control group (8\%). The size and morphology of the polyps were similar but the location differs between the groups, all HPs in the NASH group were located in the left colon, and 2/8(25\%) of HPs in the control group were located in the right colon.

The degree of liver fibrosis among the NASH group according to the liver biopsy was as follows: Fibrosis Stage 0 on 18 patients (15\%), Fibrosis Stage 1 on 29 patients $(23 \%)$, Fibrosis Stage 2 on 32 patients (26\%), Fibrosis Stage 3 on 37 patients (30\%), and Fibrosis Stage 4 on 7 patients (6\%).

It was a significant correlation between the degree of fibrosis and the prevalence of HPs as 21 from the 28 (75\%) HPs diagnosed in the NASH group were observed with high degree of fibrosis (Fibrosis Stages 3 and 4), six HPs (21\%) were associated with Fibrosis Stages 1 and 2, and a single HP (4\%) was associated with Fibrosis Stage 0.

The univariate and multivariate analyses of the risk for HPs are shown in Table 2. From the logistic regression analysis, older age $(>50)$, male sex, current smoking, high CRP levels, NASH, and advanced fibrosis were associated with increased risk for HP (Table 2).

Regarding the relationship of MS and NASH status with the presence of HP (Table 3), compared with MS negative/NASH negative group, the MS negative/NASH positive group and the MS positive/NASH positive group were significantly associated with increased risk for HPs (OR 1.3; 95\% CI, 1.02-2.13 and OR 1.48; 95\% CI, 1.05-2.04, resp.).

\section{Discussion}

This is the first study to evaluate the relationship between biopsy-proven steatohepatitis and colonic hyperplastic polyps; the results of this study demonstrate that NASH was significantly associated with an increased frequency of colonic hyperplastic polyps. Moreover, the frequency of HPs was correlated with the degree of liver fibrosis, as in patients with advanced fibrosis the possibility of having HP is higher than that in those with early or no hepatic fibrosis. Moreover, NASH and advanced hepatic fibrosis are associated with HPs independently by age, sex, smoking status, and metabolic syndrome.

Interestingly, we found a correlation between the occurrence of HPs and increased levels of C-reactive protein, as an independent predictor of HPs $[12,13]$.

A previous study reports an association between metabolic syndrome and colorectal cancer or/and colorectal adenomatous polyps $[14,15]$. Moreover, a large population based study from Korea comparing the relationship between ultrasound diagnosed NAFLD and colorectal adenomas found a direct association with NAFLD and colonic adenomas [16]. Other studies from USA found similar association between biopsy-confirmed NAFLD and the prevalence of colonic adenomas [17]. Currently, patients with more than 3 colonic adenomas or any adenoma $>10 \mathrm{~mm}$ are subject to colonoscopic screening every 3 years [18]. Thus, patients with NAFLD may need more stringent endoscopic follow-up.

NASH/NAFLD is commonly referred to as the hepatic manifestation of MS; patients with MS have been shown to have higher risks of colorectal HPs, as illustrated by several studies $[19,20]$. The mechanism that joins the two entities is most likely linked to insulin resistance (IR) that may play a distinct role in development of HPs [20, 21].

Adiponectin is an adipokine that is found in decreased concentrations not only in those who are obese and have diabetes and IR (which all is important component of the MS) but also in NAFLD/NASH patients; decreased adiponectin levels lead to increased insulin levels due to IR and increased insulin growth factor-1 (IGF-1) [22-24]. Adiponectin also directly inhibits tumor necrosis factor-alpha (TNF- $a$ ), which plays a role in tumor cell proliferation and angiogenesis (recent studies have shown that HPs may lie in the classic adenoma-carcinoma sequences and have demonstrate special molecular changes and genetic mutations which correlated with hyperplastic polyps or/and serrated colonic polyps which represent mixed feature of colonic adenomas and HPs) [25-27].

Low adiponectin levels are inversely related to colonic tumors stage and predict cancer recurrence [28, 29]. Insulin binds to IGF-1 receptors and plays an important role in cell proliferation, apoptosis, and increased production of vascular endothelial growth factor, an angiogenetic factor that supports tumor growth and may play a role in the development and growth of HPs [30].

In NAFLD, the hepatic manifestation of the MS and plasma inflammatory biomarkers are increased leading to chronic and systemic low-grade inflammatory state, mediators from the liver, including reactive oxygen species (ROS), TNF- $a$, IL-6, and PAI-1, and other proinflammatory cytokines which were positively associated with the prevalence of colorectal cancer, colonic adenomas, and most probably HPs [31]. According to our finding the combination of both NASH and MS increases the frequency of HPs due to the involvement of all the previously suggested factors.

Other interesting findings of our study are the direct correlation between hepatic fibrosis and the prevalence of 
TABLE 3: Univariate and multivariate analysis of the risk for hyperplastic polyps by NASH and metabolic syndrome.

\begin{tabular}{|c|c|c|c|c|}
\hline Variable & $\begin{array}{l}\text { Univariate analysis; } \\
\text { OR }(95 \% \mathrm{CI})\end{array}$ & Univariate analysis; $P$ value & $\begin{array}{c}\text { Multivariate analysis; } \\
\text { OR }(95 \% \mathrm{CI})\end{array}$ & Multivariate analysis; $P$ value \\
\hline MS-/NASH- & 1 & - & 1 & - \\
\hline $\mathrm{MS}+/ \mathrm{NASH}-$ & $1.48(0.94-2.34)$ & 0.193 & $1.31(1.01-1.77)$ & 0.0568 \\
\hline MS-/NASH+ & $1.62(1.27-2.03)$ & $<0.001$ & $1.30(1.02-2.13)$ & 0.034 \\
\hline $\mathrm{MS}+/ \mathrm{NASH}+$ & $1.96(1.47-2.72)$ & $<0.001$ & $1.48(1.05-2.04)$ & 0.018 \\
\hline
\end{tabular}

OR, odds ratio; CI, confidence interval; NASH, nonalcoholic steatohepatitis; MS, metabolic syndrome.

HPs; HPs are more common in advanced hepatic fibrosis and cirrhotic patients compared with early or no hepatic fibrosis; further studies are required to confirm this hypothesis and to elucidate possible mechanism responsible for the phenomenon.

Our study had several limitations; the retrospective design of the study makes it difficult to infer causality between NASH and risk for HPs. Second, there may have been a selection bias, as subjects were recruited from patients who visit the hospital (HFH) for health examination, and thus they were more concerned about their health status. Third, the population size was small to accurately reflect some known risk factors to be associated with HP as ethnicity and family history. Fourth, subjects did not undergo any molecular pathology diagnostics [32].

In conclusion, our study is the first to show an association between biopsy-proven steatohepatitis and the burden of hyperplastic polyp; the severity of hepatic fibrosis may play important role in the increase frequency of HP in those patients; further prospective studies are required to confirm the hypothesis that NASH especially with advance fibrosis may cause colorectal hyperplastic polyp.

\section{Competing Interests}

The authors declare that there is no conflict of interests regarding the publication of this paper.

\section{References}

[1] C. M. Fenoglio-Preiser, "Hyperplastic polyps, adenomatous polyps, and mixed hyperplastic adenomatous polyps of the colon: definitions," Progress in Clinical and Biological Research, vol. 279, pp. 3-12, 1988.

[2] D. K. Rex, D. J. Ahnen, J. A. Baron et al., "Serrated lesions of the colorectum: review and recommendations from an expert panel," The American Journal of Gastroenterology, vol. 107, pp. 1315-1329, 2012.

[3] C. Rosty, D. G. Hewett, I. S. Brown, B. A. Leggett, and V. L. J. Whitehall, "Serrated polyps of the large intestine: current understanding of diagnosis, pathogenesis, and clinical management," Journal of Gastroenterology, vol. 48, no. 3, pp. 287-302, 2013.

[4] S. Sweetser, T. C. Smyrk, and A. Sugumar, "Serrated polyps: critical precursors to colorectal cancer," Expert Review of Gastroenterology and Hepatology, vol. 5, no. 5, pp. 627-635, 2011.

[5] T. R. Haque, P. T. Bradshaw, and S. D. Crockett, "Risk factors for serrated polyps of the colorectum," Digestive Diseases and Sciences, vol. 59, no. 12, pp. 2874-2889, 2014.
[6] J. E. East, B. P. Saunders, and J. R. Jass, "Sporadic and syndromic hyperplastic polyps and serrated adenomas of the colon: classification, molecular genetics, natural history, and clinical management," Gastroenterology Clinics of North America, vol. 37, no. 1, pp. 25-46, 2008.

[7] M. Bertolotti, A. Lonardo, C. Mussi et al., "Nonalcoholic fatty liver disease and aging: epidemiology to management," World Journal of Gastroenterology, vol. 20, no. 39, pp. 14185-14204, 2014.

[8] M. Ahmed, "Non-alcoholic fatty liver disease in 2015," World Journal of Hepatology, vol. 7, no. 11, pp. 1450-1459, 2015.

[9] M. E. Rinella, "Nonalcoholic fatty liver disease: a systematic review," The Journal of the American Medical Association, vol. 313, no. 22, pp. 2263-2273, 2015.

[10] F. M. Mendonça, F. R. De Sousa, A. L. Barbosa et al., "Metabolic syndrome and risk of cancer: which link?" Metabolism: Clinical and Experimental, vol. 64, no. 2, pp. 182-189, 2015.

[11] E. M. Brunt, C. G. Janney, A. M. Di Bisceglie, B. A. Neuschwander-Tetri, and B. R. Bacon, "Nonalcoholic steatohepatitis: a proposal for grading and staging the histological lesions," American Journal of Gastroenterology, vol. 94, no. 9, pp. 2467-2474, 1999.

[12] R. C. Thurston, Y. Chang, C. A. Derby et al., "Abuse and subclinical cardiovascular disease among midlife women: the study of women's health across the nation," Stroke, vol. 45, no. 8, pp. 2246-2251, 2014.

[13] Y.-Z. Huang, M.-X. Li, J. Wang et al., "A 5/- regulatory region and two coding region polymorphisms modulate promoter activity and gene expression of the growth suppressor gene ZBED6 in cattle," PLoS ONE, vol. 8, no. 11, Article ID e79744, 2013.

[14] S. Riondino, M. Roselli, R. Palmirotta, D. Della-Morte, P. Ferroni, and F. Guadagni, "Obesity and colorectal cancer: role of adipokines in tumor initiation and progression," World Journal of Gastroenterology, vol. 20, no. 18, pp. 5177-5190, 2014.

[15] K. Esposito, A. Capuano, and D. Giugliano, "Metabolic syndrome and cancer: holistic or reductionist?" Endocrine, vol. 45, no. 3, pp. 362-364, 2014.

[16] S. T. Hwang, Y. K. Cho, J. H. Park et al., "Relationship of nonalcoholic fatty liver disease to colorectal adenomatous polyps," Journal of Gastroenterology and Hepatology, vol. 25, no. 3, pp. 562-567, 2010.

[17] N. T. Touzin, K. N. V. Bush, C. D. Williams, and S. A. Harrison, "Prevalence of colonic adenomas in patients with nonalcoholic fatty liver disease," Therapeutic Advances in Gastroenterology, vol. 4, no. 3, pp. 169-176, 2011.

[18] A. Castells, M. Andreu, G. Binefa, A. Fité, R. Font, and J. A. Espinàs, "Postpolypectomy surveillance in patients with adenomas and serrated lesions: a proposal for risk stratification in 
the context of organized colorectal cancer-screening programs," Endoscopy, vol. 47, no. 1, pp. 86-87, 1998.

[19] J. H. Kim, Y. J. Lim, Y. H. Kim et al., "Is metabolic syndrome a risk factor for colorectal adenoma?” Cancer Epidemiology, Biomarkers \& Prevention, vol. 16, no. 8, pp. 1543-1546, 2007.

[20] Y.-Y. Wang, S.-Y. Lin, W.-A. Lai, P.-H. Liu, and W. H.-H. Sheu, "Association between adenomas of rectosigmoid colon and metabolic syndrome features in a Chinese population," Journal of Gastroenterology and Hepatology, vol. 20, no. 9, pp. 1410-1415, 2005.

[21] W. Foltyn, B. Kos-Kudla, J. Strzelczyk et al., "Is there any relation between hyperinsulinemia, insulin resistance and colorectal lesions in patients with acromegaly?" Neuro Endocrinology Letters, vol. 29, no. 1, pp. 107-112, 2008.

[22] C. H. Tae, S.-E. Kim, S.-A. Jung et al., "Involvement of adiponectin in early stage of colorectal carcinogenesis," BMC Cancer, vol. 14, article no. 811, 2014.

[23] M. Mutoh, N. Teraoka, S. Takasu et al., "Loss of adiponectin promotes intestinal carcinogenesis in Min and wild-type mice," Gastroenterology, vol. 140, no. 7, pp. 2000-2008.e2, 2011.

[24] J. Chen and X.-F. Huang, "The signal pathways in azoxymethane-induced colon cancer and preventive implications," Cancer Biology and Therapy, vol. 8, no. 14, pp. 1313-1317, 2009.

[25] A. Kumor, P. Daniel, M. Pietruczuk, and E. Małecka-Panas, "Serum leptin, adiponectin, and resistin concentration in colorectal adenoma and carcinoma (CC) patients," International Journal of Colorectal Disease, vol. 24, no. 3, pp. 275-281, 2009.

[26] E. Erarslan, C. Turkay, A. Koktener, C. Koca, B. Uz, and N. Bavbek, "Association of visceral fat accumulation and adiponectin levels with colorectal neoplasia," Digestive Diseases and Sciences, vol. 54, no. 4, pp. 862-868, 2009.

[27] L. Norris, A. Malkar, E. Horner-Glister et al., "Search for novel circulating cancer chemopreventive biomarkers of dietary rice bran intervention in Apc mice model of colorectal carcinogenesis, using proteomic and metabolic profiling strategies," Molecular Nutrition and Food Research, vol. 59, no. 9, pp. 18271836, 2015.

[28] T. Ayyildiz, E. Dolar, N. Ugras, S. B. Adim, and O. Yerci, "Association of adiponectin receptor (Adipo-R1/-R2) expression and colorectal cancer," Asian Pacific Journal of Cancer Prevention, vol. 15, no. 21, pp. 9385-9390, 2014.

[29] S. W. Oh, C.-Y. Park, E. S. Lee et al., "Adipokines, insulin resistance, metabolic syndrome, and breast cancer recurrence: a cohort study," Breast Cancer Research, vol. 13, article R34, 2011.

[30] W. K. Al-Delaimy, S. W. Flatt, L. Natarajan et al., "IGF1 and risk of additional breast cancer in the WHEL study," EndocrineRelated Cancer, vol. 18, no. 2, pp. 235-244, 2011.

[31] P. Oset, R. Talar-Wojnarowska, A. Kumor, and E. MałeckaPanas, "Proinflammatory markers serum concentrations in patients with adenomatous colon polyps," Przeglad Lekarski, vol. 71, no. 2, pp. 78-81, 2014.

[32] S. Ogino, R. Nishihara, T. J. Vanderweele et al., "The role of molecular pathological epidemiology in the study of neoplastic and non-neoplastic diseases in the era of precision medicine," Epidemiology, vol. 27, no. 4, pp. 602-611, 2016. 


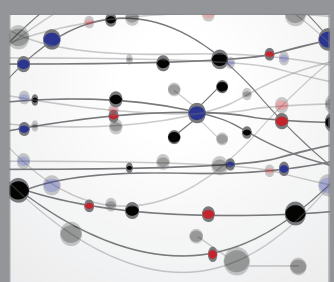

The Scientific World Journal
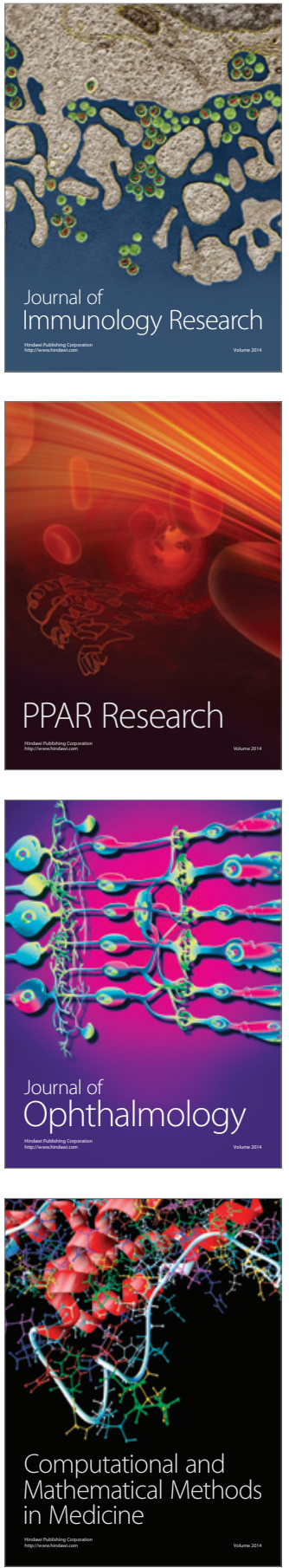

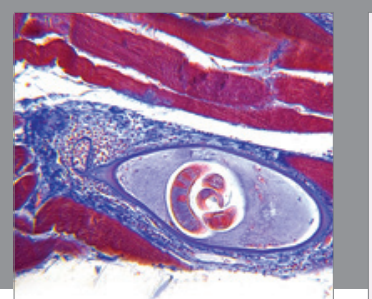

Gastroenterology Research and Practice
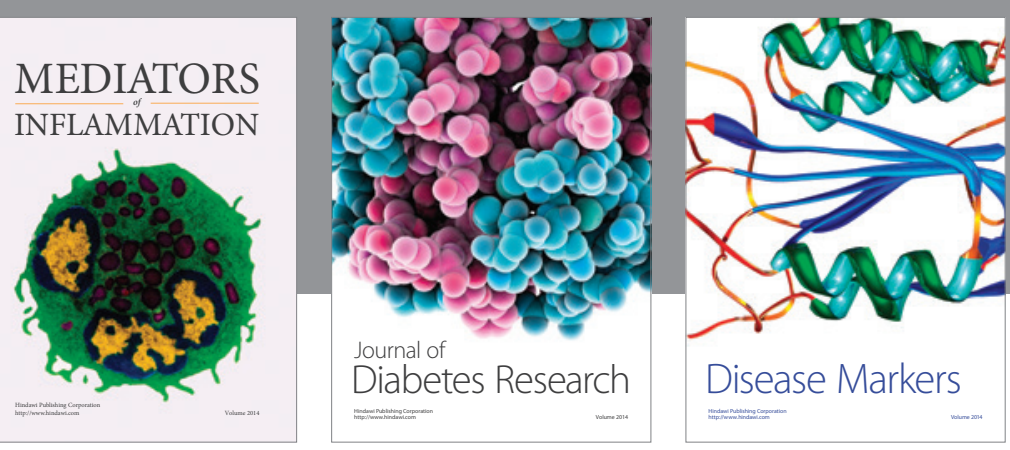

Disease Markers

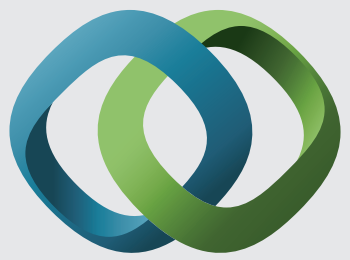

\section{Hindawi}

Submit your manuscripts at

https://www.hindawi.com
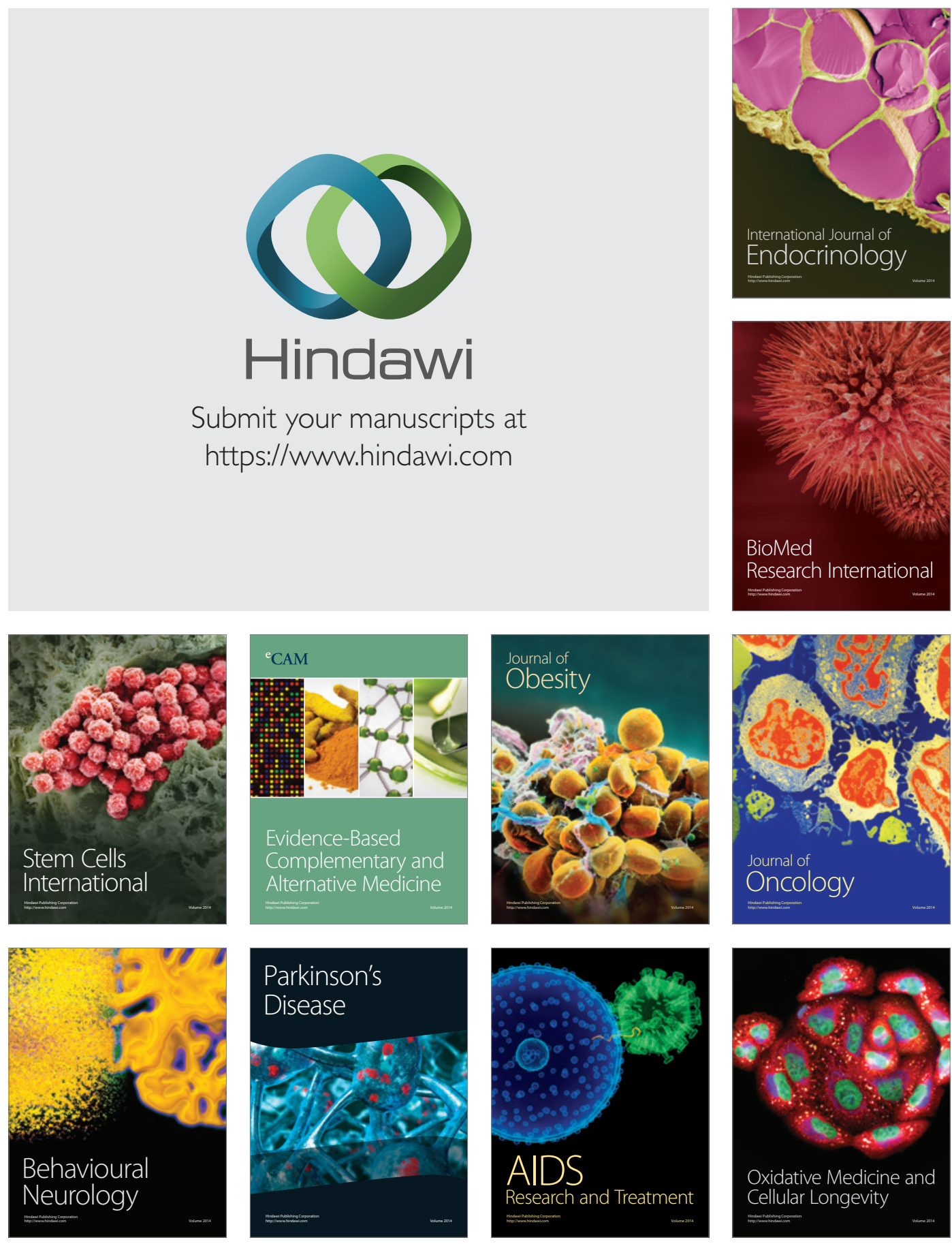